\title{
Multiple Cutaneous Metastasis of Sacral Chordoma, Mimicking Neurofibromas Clinically
}

\author{
Neha Chopra Narang*, Salil Narang and Preeti Diwaker \\ Dept. of Pathology, UCMS, Delhi, India
}

\begin{abstract}
Chordoma is a rare primary bone tumor of notochordal origin, constituting 1-4\% of malignant primary bone tumors. Chordomas are slow growing tumors and show aggressive local extension, multiple recurrences and metastasis. Cutaneous metastasis of chordoma is very rare. We report a case of a 61-year-old man presenting with multiple cutaneous metastasis of primary sacral chordoma.
\end{abstract}

Keywords: Cutaneous metastasis, Sacral Chordoma, Neurofibroma

\section{Introduction}

Chordomas are rare but aggressive tumors due to local recurrence and distant metastasis. They most commonly arise from notochordal remnants found throughout the axial skeleton. The common sites of metastasis include lungs, liver, bone, lymph nodes and rarely skin. ${ }^{[1]}$ Cutaneous involvement is usually seen because of direct extension from primary tumor or local recurrence. Distant cutaneous metastasis of chordoma is a rare event.

\section{Case Report}

A 61-year-old male was diagnosed with sacral and dorsal chordoma in November 2011. He initially complained of pain around the sacral region for 2 to 2.5 years with exaggeration of pain while sitting which subsided with oral medications. In November 2011 patient was suspected of having pilonidal sinus, however histopathological examination of specimen did not revealed any sinus tract or granuloma. MRI performed was suggestive of tumor arising in sacral region, S3 segment downwards. Patient underwent sacrectomy $\mathrm{S} 3,4,5$ with mesh reconstruction in March 2012. The gross examination revealed grey brown circumscribed tumor reaching upto bone however all the resected margins were free. Microscopic examination showed a lobulated moderately cellular tumor having cords of epithelioid tumor cells in a myxoid background reported as suggestive of chordoma. The patient was asymptomatic till December 2013. However the pain worsened in February 2014. A PET CT Scan showed active disease foci in sacrum, pelvis, mediastinal lymph nodes, vertebrae and rib. MRI showed a heterogenously enhacing mass lesion involving sacrum and coccyx with bone destruction. Patient was started on Imatinib and showed partial response to therapy. Patient also received 12 fractions of radiotherapy. D11 laminectomy with cord decompression
D10-D12 was done in January 2015. In August 2016 the patient developed low back pain radiating to both lower limbs which had aggravated over last 2-3 months. CECT revealed large lytic expansile mass lesion involving sacrum with infiltration into posterior aspects of bilateral sacro-iliac joints and anterior presacral space. Lytic lesions were also noted in D9 vertebra. Wide excision of the tumor with laminectomy was performed. Four months later the patient presented with chief complaints of intractable back pain radiating to both the lower limbs with difficulty in walking/ standing and difficulty in micturition. Multiple swellings were noted on forehead, lip and scalp, and a clinical diagnosis of neurofibromas was made. Excision biopsies of the above lesions were performed.

Histopathological findings: Gross examination showed three skin covered lesions measuring $3 \times 2 \times 1 \mathrm{~cm}, 4 \mathrm{x}$ $2 \times 1.5 \mathrm{~cm}$ and $7 \times 5 \times 3 \mathrm{~cm}$ from lip, scalp and forehead respectively. Cut surface of the lesions appeared grey-tan to pale blue and focal hemorrhagic areas were noted in the scalp lesion.

Microscopic examination revealed lobulated lesion involving the dermis. The individual lobules were separated by fibrous bands. The tumor cells were arranged in sheets cords and singly scattered in a myxoid stroma. Some tumor cells had abundant pale vacuolated cytoplasm (Physalipherous cells) while others showed eosinophilic cytoplasm. Tumor cell displayed mild to moderate nuclear atypia, however no mitosis was seen (Figure1). Histomorphological features were suggestive of cutaneous metastasis of chordoma and were confirmed using immunohistochemical markers which revealed diffuse positivity for Pan-cytokeratin and vimentin; however S-100 was negative (Figure 2). 


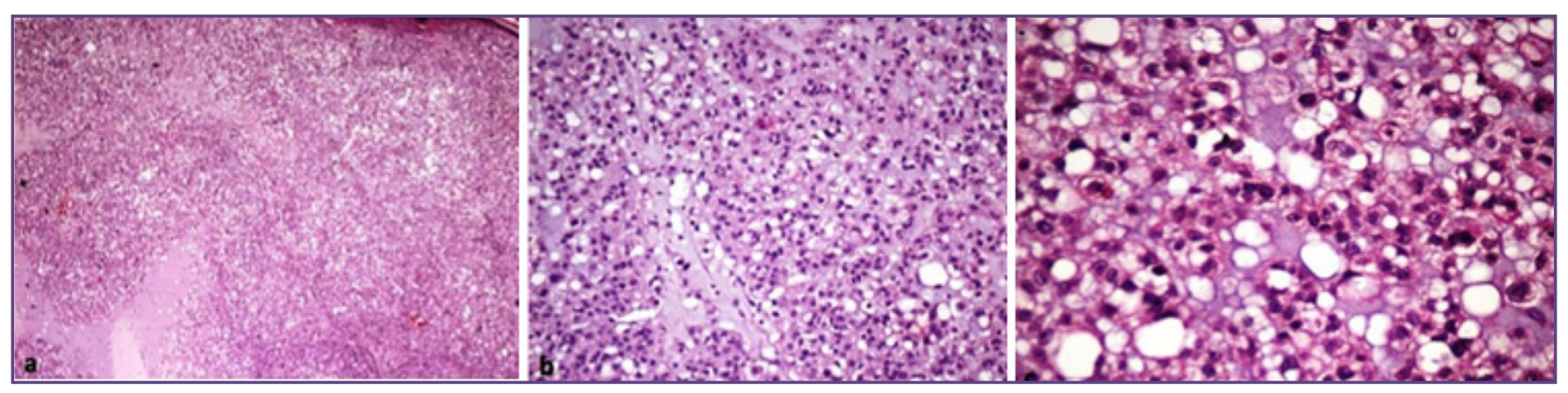

Fig. 1 a) Skin with compressed epidermis and tumor mainly involving dermis. (H\&E x40). b) Cells having clear cytoplasm with some cells with eosinophilic cytoplasm on a myxoid stroma (H\&E x100). c) Cells with abundant pale vacuolated cytoplasm (Physalipherous cells) (H\&E x 200).

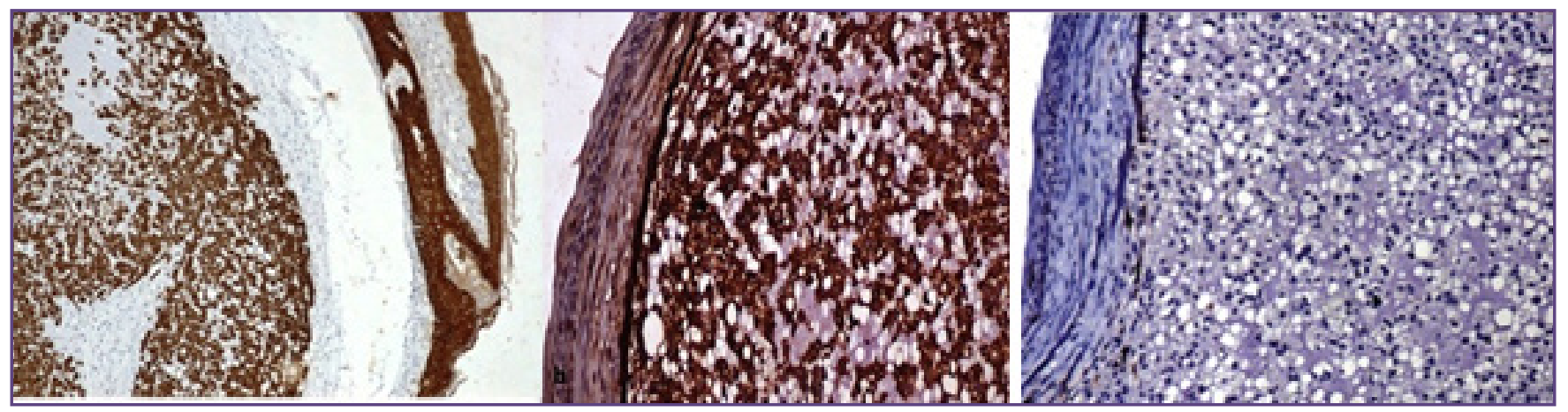

Fig. 2 Immunohistochemical staining. Skin metastasis from chordoma show a) Cytoplasmic staining with Pancytokeratin, b) Cytoplasmic staining with vimentin and negative for c) S100.(DAB x100).

\section{Discussion}

Chordoma is rare primary tumor of bone with an average incidence of 0.1 per 100,000 per year ${ }^{[1]}$. Sacrococcygeal chordomas (M:F: 2:1) usually occur during the fifth and sixth decades. ${ }^{[2]}$ The incidence of distant metastasis varies between $3 \%-50 \% \cdot{ }^{[3]}$ In a study by Patrick et al it was found that the genesis of metastasis is not related to surgical intervention, length of survival, age and sex, however the more locally aggressive chordomas which have been irradiated are more likely to metastasize. ${ }^{[3]}$

There has been an increase in the number of reported cases of cutaneous involvement of chordomas, which have been related to direct invasion or loco-regional involvement. However distant cutaneous metastasis is extremely rare.

In a retrospective study by $\mathrm{Su}$ et al, 207 cases of chordomas were reviewed out of which 9\%(19) patients had skin involvement and only 1 presented as distant skin metastasis ${ }^{[4]}$ Face or back has been found to be the most common cutaneous metastatic site, though scalp, trunk and extremities have also been reportedly involved. ${ }^{[5,6]}$

Diagnostic difficulties of metastatic chordoma are mainly due to histologic similarities with other dermal chondroid lesions. Differential diagnosis includes both primary as well as metastatic lesions having abundant mucinous/myxoid component. [Table1] ${ }^{[7]}$ Among these lesions parachordomas and extraskeletal myxoid chondrosarcomas are difficult to differentiate from chordoma. However complete clinicoradiological work up and immunohistochemical analysis is essential for definitive diagnosis, and help us to differentiate chordoma from the other lesions mentioned.

Immunohistochemistry is essential to arrive at a definitive diagnosis. Triple positivity for cytokeratin, vimentin and S-100 has proved to be a sensitive marker. ${ }^{[8,9]}$ However occasional reports have stated loss of S-100 expression in metastatic lesions ${ }^{[5,6]}$ Brachyury has emerged as a novel biomarker to differentiate chordomas from other chondroid lesions with high sensitivity and specificity when combined with cytokeratins, $98 \%$ and $100 \%$ respectively. Brachyury is a transcription factor that regulates mesoderm and notochord formation in humans. ${ }^{[10]}$

Cutaneous metastasis of chordoma even though rare should be considered as a diagnostic possibility when new skin lesions appear in a patient with chordoma. Immunohistochemistry is crucial to make a diagnosis which is usually concordant with that of the primary tumor. 
Table 1: Differential diagnosis of chordoma

\begin{tabular}{|l|}
\hline Primary tumors \\
\hline Low-grade (myxoid) chondrosarcoma \\
Myxoid liposarcoma \\
Parachordoma \\
Clear cell sarcoma \\
Myxopapillary ependymoma \\
Chondroma \\
Chondroid syringoma \\
Melanocytic lesions with balloon cell morphology \\
\hline Metastatic tumors \\
\hline Mucinous cell adenocarcinoma \\
Clear cell adenocarcinoma \\
Signet ring cell adenocarcinoma \\
\hline
\end{tabular}

\section{References}

1. Newton H. Chordoma. In: Raghavan D, Brecher ML,Johnson $\mathrm{DH}$, et al, eds. Textbooks of uncommon cancer 3rd ed. Chichester: Wiley; 2006:614-625.

2. Horwitz T. Chordal ectopia and its possible relationship to chordoma. Arch Pathol. 1941; 31:354-362.

3. Chambers PW, Schwinn CP. Chordoma: a clinicopathologic study of metastasis. Am J Clin Pathol. 1979;72:765-776.
4. Su WP, Louback JB, Gagne EJ, et al. Chordoma cutis: a report of nineteen patients with cutaneous involvement of chordoma. J Am Acad Dermatol. 1993;29:63-66.

5. Collins GR, Essary L, Strauss J, et al. Incidentally discovered distant cutaneous metastasis of sacral chordoma: a case with variation in S100 protein expression(compared to the primary tumor) and review of the literature. J Cutan Pathol. 2012;39: 637-643.

6. Ruiz HA, Goldberg LH, Humphreys TR, et al. Cutaneous metastasis of chordoma. Dermatol Surg. 2000;26:259.

7. Layfield LJ. Cytologic differential diagnosis of myxoid and mucinous neoplasms of the sacrum and parasacral soft tissues. Diagn Cytopathol. 2003;28:264-271.

8. Jambhekar NA, Rekhi B, Thorat $\mathrm{K}$, et al. Revisiting chordoma with brachiury "a new age" marker. Analysis of a validation study of 51 cases. Arch Pathol Lab Med. 2010;134: 1181-1187.

9. Gagne EJ, Su WP. Chordoma involving the skin: an immunohistochemical study of 11 cases. J Cutan Pathol. 1992;19:469-475.

10. Oakley GJ, Fuhrer K, Seethala RR. Brachyury, SOX-9, and podoplanin, new markers in the skull base chordoma vs chondrosarcoma differential: a tissue microarray-based comparative analysis. Mod Pathol. 2008;21:1461-1469.

*Corresponding author:

Dr Neha Chopra Narang, Dept. of Pathology, UCMS, Delhi, India

Phone: +91 9873449661

Email: nehac1080@gmail.com

Financial or other Competing Interests: None. 